\title{
A case of marantic endocarditis with systemic emboli in a patient with metastatic soft tissue sarcoma
}

\author{
Daniel Alexander Reikher, Mark Feldman* \\ Department of Internal Medicine, Texas Health Presbyterian Hospital, Dallas, Texas, United States
}

Received: May 19, 2019

DOI: $10.5430 /$ crim.v6n3p6
Accepted: June 23, 2019

Online Published: June 25, 2019

\begin{abstract}
Clinical manifestations of cancer can be categorized as resulting from direct tissue injury from the primary tumor, distant metastatic spread, or aberrant biological activity, also known as a paraneoplastic syndrome. Soft tissue sarcomas are a rare group of malignant tumors of mesenchymal origin which typically present with direct tissue injury, exerting their harmful potential by compression and mass effect. We describe a rare case of an occult retroperitoneal soft tissue sarcoma presenting with marantic endocarditis. To date, there is a paucity of available medical literature relating sarcoma to marantic endocarditis.
\end{abstract}

Key Words: Sarcoma, Embolic infarcts, Endocarditis, Marantic

\section{INTRODUCTION}

Sarcomas are mesenchymal neoplasms arising either from bone or soft tissue. ${ }^{[1-3]}$ Although this disease group tends to be uncommon in the spectrum of adult medicine, malignant sarcomas represent the most common type of solid tumor in pediatric oncology patients and are increasingly important to the medical community. ${ }^{[3]}$ Soft tissue sarcomas are further subclassified as deriving from muscle, tendon, fat, fibrous connective tissue, synovial tissue, blood vessels or nerve. ${ }^{[3]}$ The sheer pathological diversity of this subgroup reflects the embryonic potential of the originating malignant mesenchymal cell, holding the capacity to differentiate into a variety of tissues. This complex pathogenesis ultimately resulted in the classification of more than 100 histological sarcoma subtypes by the World Health Organization (WHO) ${ }^{[4]}$ The most common primary anatomic site is the lower extremity including the thigh, buttock and groin, while the retroperitoneum represents approximately $12 \%$ of reported primary lesions. ${ }^{[1,3]}$ Knowledge of the natural history of retroperi- toneal soft tissue sarcoma is limited. A retrospective analysis examined 117 cases of retroperitoneal sarcoma and found that only $14 \%$ of patients presented with distant metastases at the time of primary tumor resection. ${ }^{[5]}$ The lungs are the most common site of hematogenous spread of soft tissue sarcomas; however, autopsy reports also describe involvement of regional lymph nodes, bone as well as liver. ${ }^{[6-9]}$

\section{CASE PRESEntation}

A 46-year-old man presented with hypotension and acute hypoxic respiratory failure. Approximately six months earlier, the patient had been diagnosed with extensive lichens planus involving the oral mucosa, axilla and genitalia. His dermatologist initiated treatment with $60 \mathrm{mg}$ prednisone and $400 \mathrm{mg}$ cyclosporine daily. In the days preceding current his hospitalization, the patient experienced a prodrome of cough, sputum production, fevers, chills, anorexia and malaise. Bacterial pneumonia was suspected and he was given an outpatient course of amoxicillin/clavulanate; however, his symptoms

*Correspondence: Mark Feldman; Email: MarkFeldman@texashealth.org; Address: Department of Internal Medicine, Texas Health Presbyterian Hospital, Dallas, Texas, United States. 
progressed, and he presented to an outside emergency department and was then admitted to their hospital. At initial presentation, the patient was in septic shock and acute hypoxic respiratory failure, with a metabolic acidosis, acute kidney injury, and coagulopathy plus thrombocytopenia without evidence of disseminated intravascular coagulation. A chest $\mathrm{X}$-ray showed dense opacification of the left hemithorax. A chest CT scan revealed consolidation of the entire left lower lobe and a parapneuomonic effusion. An underlying lung mass could not be excluded. The patient was supported with non-invasive positive pressure ventilation, intravenous fluids, vasopressors, broad spectrum antibiotics and stress dose glucocorticoids. He was transferred to our medical center for a higher level of care with a presumed diagnosis of community-acquired pneumonia. The patient demonstrated clinical improvement after transfer to our hospital. He regained hemodynamic stability, was weaned from vasopressor dependency, achieved resolution of acute kidney injury and required only $2-3 \mathrm{~L} / \mathrm{min}$ of supplemental oxygen. The patient had active axillary and oral lichens planus, but symptomatically controlled with local anesthetics and a modified diet. Microbiological data found no respiratory pathogen on a respiratory PCR panel, and cultures of sputum and blood were negative. The patient also had negative urinary antigens for Streptococcus pneumoniae and Legionella pneumophila.

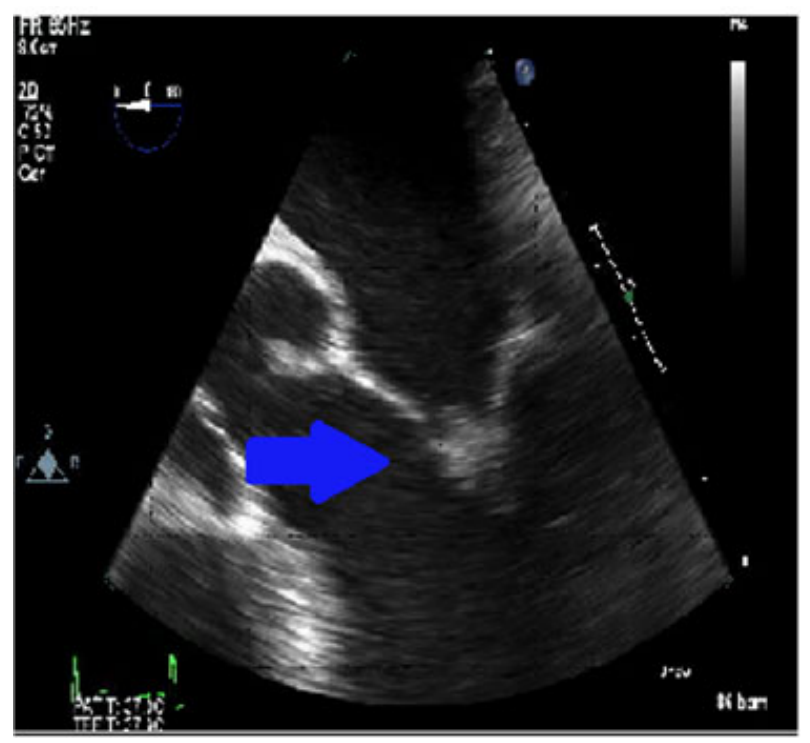

Figure 1. Transesophageal echocardiogram demonstrating a $5 \mathrm{~mm}$ mitral valve vegetation(see blue arrow)

The next day (hospital day three), the patient suddenly developed a left-sided facial droop and aphasia. A stroke code was activated and an emergent head CT scan showed no acute intracranial bleed; however, the patient was not a candidate for intravenous thrombolytic stroke therapy because of sig- nificant thrombocytopenia (platelet count, 30k). Subsequent MRI of the brain showed multiple acute cerebral infarcts, likely embolic, located in the right middle cerebral artery vascular territory. The initial transthoracic echocardiogram was negative. However, the next day, looking for a source of emboli, a $5 \mathrm{~mm}$ mitral valve vegetation was found on transesophageal echocardiography (see Figure 1) and a deep venous thrombosis (DVT) in the right common femoral vein on Doppler imaging.

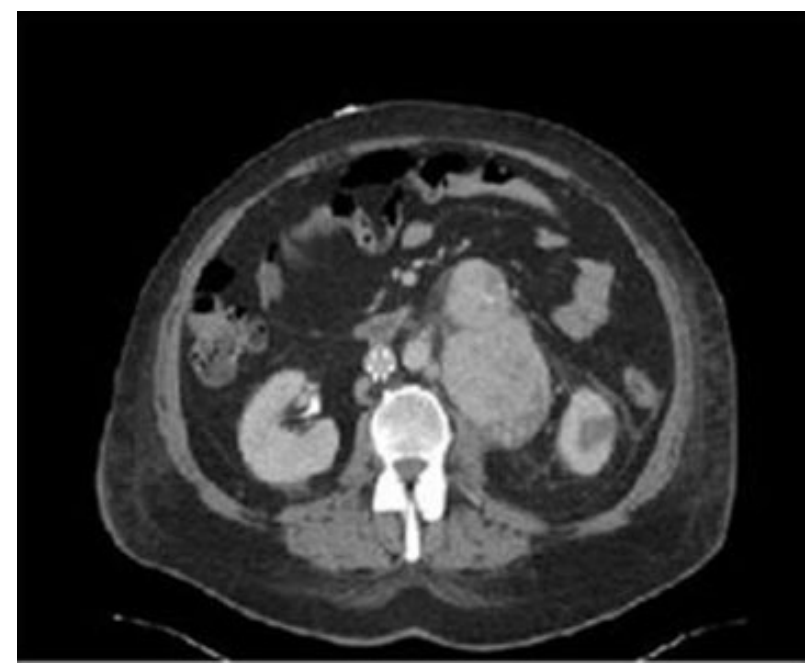

Figure 2. Lobulated masses in the left retroperitoneum and left pelvis with left renal infarct

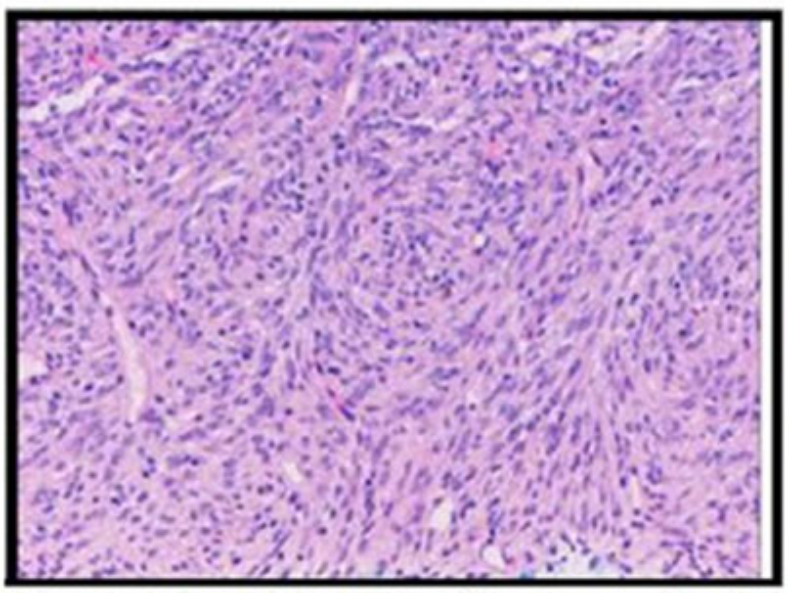

Figure 3. Biospy of left retroperitoneal mass showing malignant spindle cell neoplasm consistent with sarcoma. Hematoxylin/eosin stain

Hyperbilirubinemia, rising to $4 \mathrm{mg} / \mathrm{dl}$ developed after this patient's embolic strokes. Therefore, abdominal ultrasonography was obtained, which showed no biliary tract or hepatic disease, but revealed an adjacent abdominal mass. A subsequent abdominal and pelvic CT further characterized a 
large retroperitoneal mass measuring $8 \mathrm{~cm} \times 6 \mathrm{~cm} \times 4.5 \mathrm{~cm}$, as well as splenic and renal infarcts, presumably embolic (see Figure 2). The patient underwent interventional radiologyguided biopsy of the retroperitoneal mass and a diagnosis of malignant spindle cell sarcoma with a low to moderate proliferative index was established on pathology (see Figure 3). Systemic chemotherapy with daunorubicin and ifosfamide was instituted. He received two cycles, but tolerated the therapy poorly, with chemotherapy-associated nausea and severe mucositis. His disease progressed as he developed a metastatic lesion in the right hip abductor musculature, as seen on repeat CT scan. He was readmitted with acute kidney injury within two months of initial diagnosis. The patient and family then decided to pursue comfort care, and he passed away soon thereafter.

\section{Discussion}

This patient had mitral valve endocarditis with subsequent cerebral, splenic and renal embolization. The defining element in the pathogenesis of endocarditis is the formation of a valvular vegetation, composed mainly of fibrin and platelets. ${ }^{[10]}$ Vegetations are then further classified as either infectious (bacterial, fungal) or noninfectious, the latter commonly known as nonbacterial thrombotic or marantic endocarditis. While marantic endocarditis is most commonly associated with cancer, there is also a well-established association with autoimmune-mediated diseases such as systemic lupus erythematosus (SLE), antiphospholipid syndrome and rheumatoid arthritis. ${ }^{[11]}$ In this patient with sarcoma and mitral valve vegetations, two sets of blood cultures for bacterial and fungal organisms were negative, supporting a diagnosis of marantic endocarditis. Infectious endocarditis cannot be fully ruled out as the patient's prior antibiotic exposure decreased the negative predictive value of sterile blood cultures. A case series published in 1976 examined 65 autopsy cases of marantic endocarditis. Fifty one $(78 \%)$ of those cases were found to have an associated malignancy, with pancreas, lung, stomach and adenocarcinoma of unknown primary being the most common cancers. ${ }^{[12]}$ To our knowledge, soft tissue sarcoma has not been previously reported in association with marantic endocarditis. ${ }^{[12-14]}$ The underlying pathophysiology of marantic endocarditis is thought to be driven by acquired hypercoagulability and a systemic proinflammatory state. ${ }^{[14]}$ The association between cancer and thrombophilia has been long described, dating back the original work of Virchow and Trousseau in the 19th century. ${ }^{[14,15]}$ Cancer propagates a hypercoagulable state both by direct and indirect pathways. Direct pathways include procoagulant expression by tumor cells, an aberrant host inflammatory response and cytokine release. Indirect pathways include direct vascular injury by the mass, in addition to the iatrogenesis of surgery, chemotherapy and immobility. ${ }^{[14]}$ Circulating cytokines are hypothesized to initiate valvular damage, compromising the natural endothelial valve surface. This damage triggers overactive platelet and fibrin deposition, eventually forming a vegetation. ${ }^{[13,14]}$ Animal models of marantic endocarditis further describe the association between circulating tissue factor (TF), TF mRNA expressivity by valvular monocytes and the presence of a cardiac lesion. ${ }^{[16]}$ The histology and complications of marantic endocarditis are best understood in a subset of autoimmune associated cases known as Libman-Sacks endocarditis. A comprehensive case series from the Mayo Clinic examined 30 reports of Libman-Sacks endocarditis between 1985 to 2000. Pathological examination found that the vegetations were primarily composed of fibrin and platelets, with minimal inflammatory cells and absent bacteria. ${ }^{[17]}$ This distinct composition has led experts to believe that vegetations in marantic endocarditis are more friable and likely to dislodge, increasing the probability for systemic embolization. ${ }^{[14,17]}$ Additional reports further demonstrated an association between neuropsychiatric SLE and the presence of Libman-Sacks vegetations. ${ }^{[11]}$ The main clinical manifestation of marantic endocarditis is systemic embolization, as in our patient. Previous studies also described valve dysfunction (insufficiency, stenosis) as a potential consequence, but were unable to establish a temporal relationship as to whether valvular dysfunction was a stimulus for or a consequence of valvular vegetation. ${ }^{[17]}$ Common sites of embolization include kidneys, spleen and extremities; however, central nervous system involvement is most likely to become clinically apparent, as in our patient. ${ }^{[14,17]}$ The patient's initial presentation of pneumonia, sepsis and hypoxemic respiratory failure was likely a consequence of his immunocompromised state. The patient's therapy for lichens planus included high doses of cyclosporine and prednisone, which predisposed to an increased risk of infection. The patient's daily dose of $400 \mathrm{mg}$ of cyclosporine, when accounted for body mass, is comparable to a typical dose used in organ transplantation. Calcineurin inhibitors have been linked with certain bacterial, viral and fungal infections in this patient population. ${ }^{[18]}$ Systemic glucocorticoids increase susceptibility to infection by directly suppressing the cellular immune system and secondarily by predisposing to diabetes mellitus and skin breakdown. A comprehensive, nation-wide, metaanalysis examined several cohorts of patients requiring long term prednisone therapy for rheumatologic, dermatologic and gastrointestinal autoimmune diseases. The cumulative data found a strong dose dependent association between treatment with systemic glucocorticoids and development of infection. ${ }^{[19]}$ Our patient's immunosuppressive regimen was 
potent and chronic, likely predisposing him to a severe case of an acute bacterial pneumonia, although we are unable to discern the specific pathogen, perhaps due to recent outpatient antibiotic therapy. There is no evidence supporting a casual association between malignancy and lichen planus. Although oral lichen planus can rarely undergo malignant transformation into oral cancer, no study has yet described the development of lichen planus as a paraneoplastic manifestation of an overt cancer. ${ }^{[20,21]}$ Therefore, the patients pre-existing skin condition was more likely idiopathic and unrelated to his later diagnosis of soft tissue sarcoma.

\section{Conclusion}

We describe a case initially involving an immunocompromised patient presenting with severe pneumonia. An acute neurological deficit found on a routine physical exam ultimately led to the discovery of embolic infarctions in several organs from marantic endocarditis due to a soft tissue retroperitoneal sarcoma. This unique case report deepens the body of medical knowledge by illustrating both the versatility and virulence of a heterogeneous and rare group of soft tissue malignancies.

\section{REFERENCES}

[1] Lawrence WJr, Donegan WL,Natarajan N. Adult soft tissue sarcomas. A pattern of care survey of the American College of Surgeons. Ann Surg. 1987; 205(4): 349. PMid:3566372. https://doi .org/10.1 097/00000658-198704000-00003

[2] Madabhavi I, Kataria P. Primary mediastinal synovial sarcoma presenting as superior vena cava syndrome: A Rare Case Report and Review of the Literature. Case Rep Oncol Med. 2015; 2015: 651813. PMid:26101678. https://doi.org/10.1155/2015/651813

[3] National Comprehensive Cancer Network. Soft tissue sarcoma (Version 2.2019).

[4] Fletcher CDM, Bridge JA, Hogendoorn P et al. World Health Organization Classification of Tumors of Soft Tissue and Bone, 4th ed, IARC Press, Lyon 2013.

[5] Alldinger I, Yang Q, Pilarsky C, et al. Retroperitoneal soft tissue sarcomas: prognosis and treatment of primary and recurrent disease in 117 patients. Anticancer Res. 2006 Mar-Apr; 26(2B): 1577-81.

[6] Huang MN, Edgerton F, Takita $H$, et al. Lung resection for metastatic sarcoma. Am J Surg. 1978; 135: 804-806. https : //doi .org/10 .1016/0002-9610(78) 90170-8

[7] Creagan ET, Fleming TR, Edmonson JH, et al. Pulmonary resection for metastatic non-osteogenic sarcoma. Cancer. 1979; 44 : 1908-1912 https://doi.org/10.1002/1097-0142(197911)4 4:5<1908: :AID-CNCR2820440553>3.0. CO;2-2

[8] Huth JF, Holmes EC, Vernon SE, et al. Pulmonary resection for metastatic sarcoma. Am J Surg. 1980; 140: 9-16. https ://doi .or g/10.1016/0002-9610(80)90411-0

[9] Vezeridis M, Moore R, Karakousis CP, et al. Metastatic patterns of soft tissue sarcoma. Arch Surg. 1983; 118: 915-8. PMid:6307217. ht tps://doi.org/10.1001/archsurg.1983.01390080023007

[10] Sullam PM, Drake TA, Sande MA. Pathogenesis of endocarditis. Am J Med. 1985 Jun 28; 78(6B): 110-5. https : //doi .org/10.1016/ 0002-9343 (85) 90373-0

[11] Roldan CA, Sibbitt WL, Qualls CR. Libman-Sacks endocarditis and embolic cerebrovascular disease. JACC Cardiovasc Imaging. 2013 Sep; 6(9): 973-83. PMid:24029368. https ://doi .org/10.1016/ j.jcmg. 2013.04.012
[12] Deppisch LM, Fayemi AO. Non-bacterial thrombotic endocarditis: Clinicopathologic correlations. Am Heart J. 1976; 92: 723-729 https ://doi.org/10.1016/S0002-8703(76)80008-7

[13] Mazokopakis EE, Syros PK, Starakis IK. Nonbacterial thrombotic endocarditis (marantic endocarditis) in cancer patients. Cardiovasc Hematol Disord Drug Targets. 2010; 10(2): 84-6. PMid:20397972. https://doi.org/10.2174/187152910791292484

[14] El-Shami K, Griffiths E, Streiff M. Nonbacterial thrombotic endocarditis in cancer patients: pathogenesis, diagnosis, and treatment. Oncologist. 2007 May; 12(5): 518-23. PMid:17522239. https : //doi.org/10.1634/theoncologist.12-5-518

[15] Kumar D, Hanlin E, Glurich I, et al. Virchow's contribution to the understanding of thrombosis and cellular biology. Clin Med Res. 2010 Dec; 8(3-4): 168-172. PMid:20739582. https ://doi.org/ $10.3121 / \mathrm{cmr} .2009 .866$

[16] Nakanishi K, Tajima F, Nakata Y et al. Tissue factor is associated with the nonbacterial thrombotic endocarditis induced by a hypobaric hypoxic environment in rats. Virchows Arch. 1998; 433: 375-379. PMid:9808440. https://doi.org/10.1007/s004280050262

[17] Eiken PW, Edwards WD, Tazelaar HD, et al. Surgical pathology of nonbacterial thrombotic endocarditis in 30 patients, 1985-2000. Mayo Clin Proc. 2001; 76: 1204-12. PMid:11761501. https: //doi.org/10.4065/76.12.1204

[18] Fishman JA. Infection in Organ Transplantation. Am J Transplant. 2017 Apr; 17(4): 856-879. PMid:28117944. https ://doi.org/10 .1111 /ajt. 14208

[19] Grijalva CG, Chen L, Delzell E, et al. Initiation of tumor necrosis factor- $\alpha$ antagonists and the risk of hospitalization for infection in patients with autoimmune diseases. JAMA. 2011; 306: 2331-2339. PMid:22056398. https://doi.org/10.1001/jama.2011.1692

[20] Halonen P, Jakobsson M, Heikinheimo O, et al. Cancer risk of Lichen planus: A cohort study of 13,100 women in Finland. Int J Cancer. 2018 Jan 1; 142(1): 18-22. PMid:28857160. https : //doi.org/10.1002/ijc.31025

[21] Ganesh D, Sreenivasan P, Öhman J et al. Potentially malignant oral disorders and cancer transformation. Anticancer Res. 2018 Jun; 38(6): 3223-3229. PMid:29848669. https://doi.org/10.21873/ant icanres. 12587 